\title{
Trans-Iliac Rat Aorta Stenting: A Novel High Throughput Preclinical Stent Model for Restenosis and Thrombosis
}

\author{
Shizu Oyamada, M.D. $1,{ }^{*}$, Xiaodong Ma, Ph.D. $2,3,{ }^{*}$, Tim Wu, M.D. $2,3,{ }^{\star *}$, Michael P. Robich, \\ M.D. ${ }^{1}$ Hao Wu, M.S. ${ }^{3}$, Xingwei Wang, Ph.D. ${ }^{2}$, Bryan Buchholz, Ph.D. ${ }^{2}$, Stephen McCarthy, \\ Ph.D. ${ }^{2}$, Cesario F. Bianchi, M.D. ${ }^{1}$, Frank W. Sellke, M.D. ${ }^{1}$, and Roger Laham, M.D. ${ }^{4, *}$ \\ 1 Division of Cardiothoracic Surgery, Brown Medical School, Providence, RI 02903, USA \\ 2 Biomedical Engineering and Biotechnology Doctoral Program, University of Massachusetts, \\ Lowell, MA 01854, USA \\ 3 VasoTech, Inc., Lowell, MA 01854, USA \\ ${ }^{4}$ Department of Cardiology, Beth Israel Deaconess Medical Center, Harvard Medical School, \\ Boston, MA 02215, USA
}

\begin{abstract}
Background-Currently, preclinical stent development requires elaborate large animal models which are time consuming and expensive. We herein report a high throughput rat aorta stenting model which could provide a rapid and low-cost platform for preclinical stent development.

Methods-A total of 86 metal stents (316L stainless steel 13mm, VasoTech, Inc.) coated with poly (D, L-lactide -co-glycolide) / amorphous calcium phosphate (PLGA/ACP) copolymer were pre-mounted on $1.5 \mathrm{~mm} \times 15 \mathrm{~mm}$ balloon catheters and were implanted into aspirin treated Sprague-Dawley rats (500-700g) initially using either direct placement in the abdominal aorta (Group A, $\mathrm{n}=7$ ) or a trans-iliac approach (cut-down, Group B, $\mathrm{n}=79$ ). The surviving rats were sacrificed at 1 week, 2 weeks, 4 weeks and 12 weeks post implantation and the stented arteries were analyzed histopathologically
\end{abstract}

Results-Four rats died in group A and nine rats died in group B within 48 hours post stent implantation (mortality: $57 \%$ vs. $11 \%, \mathrm{P}<0.05$ ). All animals that died had stent thrombosis/ paralysis with visible thrombus on necropsy. Histologically, neointimal growth peaked at approximately 4 weeks post implantation.

Conclusion-This result suggests that human-sized stents can be successfully implanted into the rat aorta via iliac artery insertion with a significantly higher survival rate than trans-aorta implantation. The model system allows rapid (4-12 weeks) assessment of stent biocompatibility with mortality/paralysis used as an indicator of stent thrombosis.

\section{Keywords}

Iliac artery; Aorta; Stent; Restenosis; Thrombosis

\footnotetext{
(C) 2010 Elsevier Inc. All rights reserved.

** Corresponding authors: Tim Wu. Tel: +1-617-686-2770. Fax: +1-617-446-1518. tiangenwu@ yahoo.com Roger Laham. Tel: +1-617-632-9204. Fax: +1-617-975-5201. rlaham@ caregroup.harvard.edu..

Equal contribution.
}

Publisher's Disclaimer: This is a PDF file of an unedited manuscript that has been accepted for publication. As a service to our customers we are providing this early version of the manuscript. The manuscript will undergo copyediting, typesetting, and review of the resulting proof before it is published in its final citable form. Please note that during the production process errors may be discovered which could affect the content, and all legal disclaimers that apply to the journal pertain. 


\section{Introduction}

In-stent restenosis (ISR) has been one of the most serious complications since the introduction of stent technology (1). Though the development of drug-eluting stents (DESs) was a major breakthrough as a potential solution for ISR, ISR in high risk patients with small vessels, diabetes, and long segments of diffusely diseased arteries still remains unacceptably high (30\%-60\% in bare metal stents and 6\%-18\% in drug coated stents) despite DES implantation (2-4). Therefore, for the development of next generation of DESs, a high throughput animal model is essential for stent evaluation before clinical trials.

The rabbit iliac artery and the porcine coronary models of ISR are widely used for stent evaluation, however, these models are costly with numerous well documented limitations (5). Stenting of the rat carotid artery or aorta as a model to evaluate stents has also been reported extensively (6-9). However, due to the limitation of rat carotid artery size, a specially designed miniature stent with delivery system is required, and therefore the data generated from this system are compromised. For the rat aorta stenting model in which some preliminary results on the successful coronary stent placement have been reported (10-12), as the size of rat thoracic arteries is close to that of the human coronary artery (approximately $2.5 \mathrm{~mm}$ ), a human sized stent and delivery system can be utilized. Currently, two approaches to rat aorta stent placement have been reported: through the carotid artery and direct abdominal aortic insertion. Insertion through the carotid artery to the aorta will not block blood flow, which avoids aortic thrombosis, but the procedure requires a highlyskilled physician to successfully complete. Insertion through an incision in the lower abdomen, although technically easy, requires interruption of the aortic blood flow which may cause acute thrombosis. In this study, we present a novel approach to abdominal aortic stenting utilizing the common iliac artery which results in minimal interruption in aortic blood flow. Additionally, animal survival rates were compared between our approach and direct abdominal aortic incision stenting.

\section{Materials and Methods}

\section{Animal Protocol}

This study was approved by the Institutional Animal Care and Use Committee (IACUC) of the Beth Israel Deaconess Medical Center, Harvard Medical School and performed in accordance with the protocol approved by IACUC. Specific pathogen-free, male SpragueDawley rats (Charles River, Wilmington, MA) weighing 500-700g were fed aspirinincorporated food $(5 \mathrm{mg} / \mathrm{kg} /$ day, Bio-Serv, Frenchtown, NJ) commencing three days before the surgery and maintained through the entire study period. All polymer coated stents $(1.5 \mathrm{~mm} \times 13 \mathrm{~mm})$ were pre-mounted on VasoTech ${ }^{\circledR}$ miniature balloon catheters $(1.5 \mathrm{~mm}$ $\times 15 \mathrm{~mm}$, VasoTech, Inc.) and sterilized with Ethylene Oxide (ETO) for 60 minutes before implantation.

All animals were fasted for 12 hours before the surgery. After the animal was fully anesthetized with constant inhalation of a mixture of oxygen/isoflurane (1.5:2 pressure/ pressure) and heparinized based on the animal weight with a standard dose (100 IU/kg IM) of heparin, the abdominal aorta and left iliac artery were exposed. The stents were inserted into the abdominal aorta $10 \mathrm{~mm}$ above the bifurcation through either abdominal aorta incision (Group A, $n=7$ ) or left iliac arterial incision (Group B, $n=79$ ) by arteriotomy and direct placement, and were deployed by inflating the balloon catheter to 10ATM pressure for 30 seconds. The balloon catheter was deflated to maintain negative pressure for 30 seconds. The process was repeated three times to fully deploy the stent. The deflated catheter was then withdrawn slowly while leaving the stent in place. The arteriotomy was 
then repaired with 7-0 bio-absorbable suture. All animals were allowed to recover and returned to the animal care facility where they continued receiving antiplatelet therapy for the entire study period. Antibiotics (Gentamycin, $50 \mathrm{mg} / \mathrm{kg}$, IM, Abbott Laboratories, Abbott Park, Chicago, IL) were given to all animals for three days following the surgery. Postoperative analgesia was administrated, as needed, at the discretion of the attending veterinarian.

Animals were monitored daily with body weight changes and signs of thrombosis (paralysis of lower extremities) post stent implantation through the entire study period. If there was any sign of thrombosis, the animals were scarified and necropsied immediately. The surviving rats were sacrificed at predetermined time points of 1,2, 4 and 12 weeks. During the necropsy, the rats were first perfused with heparinized saline under deep anesthesia at $80 \mathrm{mmHg}$ pressure through the left ventricle until the perfusate from right atria was clear of blood and were then continuously perfused with $4 \%$ buffered formalin for 30 minutes to fix the stented arteries. The stented arteries were then removed and stored in $4 \%$ buffered formalin for further pathological processing. For the pathological analysis, the stented arterial tissue was plastic embedded, processed and stained with Hematoxylin \& Eosin (H \& E) as previously described by Quentin et al (13). Briefly, the fixed stented arteries were dehydrated and embedded in methylmethacrylate (Technovit 9100 New kit, Heraeus Kulzer GmbH, Germany) following the protocol supplied by the manufacturer. Three $200 \mu \mathrm{m}$-thick cross-sections were cut from the proximal, middle and distal points of the resin-embedded specimen using a low-speed precision saw (IsoMet 1000, Buehler, IL). All sections were then ground down to approximately $10-20 \mu \mathrm{m}$ using silicon carbide abrasive papers with decreasing grit (320/600/1200) on a variable speed grinder-polisher (EcoMet 3000, Buehler, IL). The polished cross-section was rehydrated and stained with Hematoxylin \& Eosin (Fisher Scientific Company, LLC, Kalamazoo, MI).

The morphopathological analysis was completed by an investigator blinded to the treatment groups. Each section was examined under a microscope (Olympus BX60, Olympus Companies, Japan) with a camera system (Hitachi HV-C20 3-CCD Color Camera, Hitachi Densi, Ltd, Japan) and computerized histomorphometrically by an image analysis system (Image-Pro® Plus Version 4.5, Media Cybernetics, Inc., Bethesda, MD). The cross sectional areas of the lumen, neointima, media and adventia were measured. Percentage of restenosis was calculated as follows: The area within the Internal Elastic Lamina (IEL) was considered the normal lumen area. The IEL area and residual area were traced using a light microscope image system as described before. The percentage of new intima (restenosis) will be defined as [(IEL area-Residual Lumen Area)/IEL] $\times 100$. Neointima area was determined by subtracting the area of the residual lumen from the area within the internal elastic lamina. The mean measured value of three points (the proximal, the middle and the distal) was calculated as the final measurement of each specimen.

\section{Statistical Methods}

All measurements were expressed as mean $+/-$ standard deviation (SD). The differences between the groups were tested for the statistical significance using ANOVA followed by Tukey's post-hoc test. Difference in mortality rates between groups was determined by $\chi^{2}$ or Fisher's exact test. A P value less than 0.05 was considered statistically significant.

\section{Results}

\section{Acute Thrombosis Rate}

In group A, a total of 7 stents were successfully deployed, but 4 rats died due to acute thrombosis, while in group B, a total of 79 stents were successfully deployed with 9 rat 
deaths due to the acute thrombosis (Mortality: $57 \%$ vs. $11 \%, \mathrm{P}<0.05$, Fig. 1). Twenty four rats selected randomly from the surviving rats were successfully harvested and examined at the following time points: 1 week $(n=6), 2$ weeks $(n=6), 4$ weeks $(n=6), 12$ weeks $(n=6$, one stent lost). Fig. 2 is the schematic illustration of two different surgical methods and Fig. 3 is the operative photographs of trans-iliac artery surgery.

\section{Histomorphometric Analysis}

Fig. 4 is the chart showing the morphometric analysis of neointimal growth in surviving animals. As shown in the chart, the neointima increased fast during first 4 weeks and peaked at approximately 4 weeks implantation. (Note: $\mathrm{P}<0.05$ between 1 week and 4 weeks, but $\mathrm{P}>0.05$ between 4 weeks and 12 weeks.)

Histopathologically, no thrombus was found inside the stented arteries of any of the surviving animals 48 hours after implantation. The neointimal growth started at 1 week (Fig. $5 \mathrm{~A}$ ), gradually invaded the space between the stent struts at 2 weeks (Fig. 5B), and peaked at approximately 4 weeks post implantation (Fig. 5C). By 12 weeks post implantation, the inner layer of the stented arterial walls was filled with "healed scar" tissue with a layer of endothelial cells at the top (Fig. 5D). Fig. 5 shows the representative images taken from those time points.

\section{Discussion}

Although rat aorta stenting has been used widely for the development of stent related products, we believe that we are the first to report the placement of human-sized stents in the rat aorta through the common iliac artery. In the study, we compared the survival rate between the two different approaches (trans-aorta vs. trans-iliac artery) and found that there was a significant difference between them. Necropsy findings showed that acute thrombosis was the major cause of the death including: 1) thrombosis in the stented artery. The acute thrombosis was likely to occur around the deployed stent even in the heparinized state, particularly during flow interruption for proximal vascular control followed by balloon occlusion for stent deployment in the tans-aortic incision. In contrast, in the trans-iliac incision group the surgical procedure was performed without interruption in blood flow while gaining vascular control, and therefore thrombosis occurred rarely in the aorta. This may be secondary to reduced vessel injury that inevitably occurs while circumferentially dissecting the aorta and physically constricting it; 2) thrombosis at the incision/suture site. In the trans-iliac arterial stenting model, as the stent was inserted through the iliac artery, the main aortic trunk remained uninjured. Therefore, paralysis of the bilateral limbs was avoided as was acute thrombosis in the abdominal aorta. We feel avoiding the aortotomy is one key advantage of this procedure.

The study also showed that the morpho-pathological change of the stented artery was not significantly different from that reported with the aortic stenting model through traditional direct aortic incision and delivery approach. The results indicate that the trans-iliac aortic stenting model system is comparable to other aorta stenting models and therefore is reliable for data interpretation.

For increasing animal survival rate, several technical points are worth emphasizing here: 1) since the vessel wall of rat vena cava and the common iliac vein are extremely thin and are very easy to injure, resulting in bleeding under heparinization conditions, the physician should pay particularly attention to avoid injury of these two veins; 2 ) there will be a learning curve for an operator before survival rates are stable. Generally speaking, practice of 2-3 rat surgeries is needed to make the physician familiar with the technique and the 
whole surgery protocol; 3) always use bigger animals, 500 grams or more. The bigger the animal is, the easier to insert the stent into the artery.

However, several limitations of the study should be pointed out here: 1) due to the budget limitation and particular study situation, the rat number of group A is statistically low; 2) all rats in the study were heparinized based on their weight with a standard dose (100 IU/kg IM), which may cause the rats underdosed or overdosed.

\section{Conclusion}

In conclusion, human sized stents can be inserted into the rat aorta through the trans-iliac artery with less acute thrombosis and mortality, and therefore the trans-iliac artery stenting model is a reliable model system for the high throughput of DES studies.

\section{References}

1. van der Hoeven BL, Pires NMM, Warda HM, Oemrawsingh PV, van Vlijmen BJM, et al. Drugeluting stents: results, promises and problems. Int J Cardiol. 2005; 99:9-17. [PubMed: 15721493]

2. Iijima R, Ikari Y, Miyazawa A, Nakajima H, Hara K. Predictors of restenosis after implantation of $2.5 \mathrm{~mm}$ stents in small coronary arteries. Circ J. 2004; 68:236-240. [PubMed: 14993779]

3. Schofer J, Schlüter M, Gershlick AH, Wijns W, García E, et al. Sirolimus-eluting stents for treatment of patients with long atherosclerotic lesions in small coronary arteries: double-blind, randomised controlled trial (E-SIRIUS). Lancet. 2003; 362:1093-1099. [PubMed: 14550694]

4. Muramatsu T, Tsukahara R, Ho M, Ito Y, Ishimori H, et al. Clinical outcome of stent implantation in small coronary arteries using different type of coronary stents. J Invasive Cardiol. 2001; 13:634639. [PubMed: 11533501]

5. Johnson GJ, Griggs TR, Badimon L. The utility of animal models in the preclinical study of interventions to prevent human coronary artery restenosis: on behalf of the subcommittee on animal, cellular and molecular models of thrombosis and haemostasis of the scientific and standardization committee of the international society of thrombosis and haemostasis. Thromb Haemost. 1999; 81:835-843. [PubMed: 10365761]

6. Fishbein I, Alferiev I, Bakay M, Stachelek SJ, Sobolewski P, et al. Local delivery of gene vectors from bare-metal stents by use of a biodegradable synthetic complex inhibits in-stent restenosis in rat carotid arteries. Circulation. 2008; 117:2096-2103. [PubMed: 18413497]

7. Indolfi C, Esposito G, Stabile E, Cavuto L, Pisani A, et al. A new rat model of small vessel stenting. Basic Res Cardiol. 2000; 95:179-185. [PubMed: 10879619]

8. McMahon AC, Zreiqat H, Lowe HC. Carotid artery stenting in the Zucker rat: a novel, potentially 'diabetes-specific' model of in-stent restenosis. Diab Vasc Dis Res. 2008; 5:145-146. [PubMed: 18537104]

9. Vermeersch P, Nong Z, Stabile E, Varenne O, Gillijns H, et al. L-arginine administration reduces neointima formation after stent injury in rats by a nitric oxide-mediated mechanism. Arterioscler Thromb Vasc Biol. 2001; 21:1604-1609. [PubMed: 11597933]

10. Lowe HC, Chesterman CN, Khachigian LM. Rat aortic stenting: toward a simple model of in-stent restenosis. Am J Cardiol. 2001; 88:720-721. [PubMed: 11569492]

11. Roks AJM, Henning RH, van Boven AJ, Tio RA, van Gilst WH. Rat abdominal aortic stenting: a simple model displaying in-stent restenosis. Am J Cardiol. 2002; 89:1149-1150. [PubMed: 11988215]

12. Lowe HC, James B, Khachigian LM. A novel model of in-stent restenosis: rat aortic stenting. Heart. 2005; 91:393-395. [PubMed: 15710738]

13. Quentin T, Poppe A, Bär K, Sigler A, Foth R, et al. A novel method for processing resin-embedded specimens with metal implants for immunohistochemical labelling. Acta Histochem. 2009; 111:538-542. [PubMed: 18554687] 


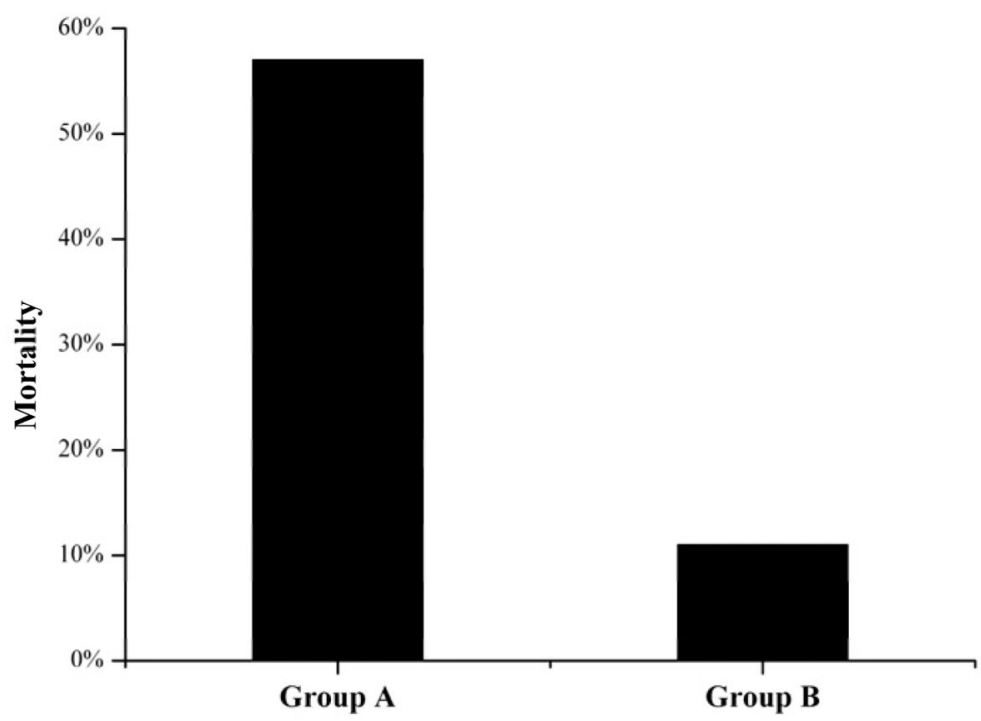

Fig. 1.

Animal mortality of two surgical methods: Trans-abdomial aorta (Group A, n=7) and Trans-iliac artery (Group B, $\mathrm{n}=79$ ) 


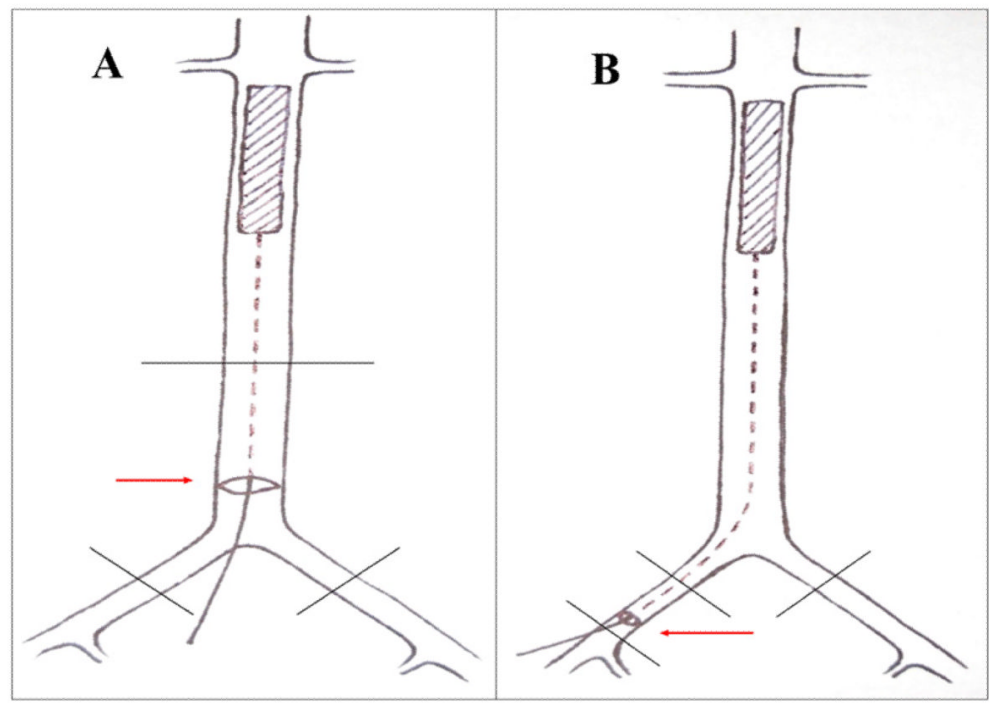

Fig. 2.

Schematic pictures of A. Trans-abdominal aorta and B. Trans-iliac artery. Arrows indicate the aortic/arterial incisions. 


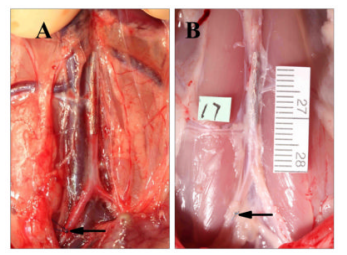

Fig. 3.

Operative photographs of rat abdominal aorta stenting through left iliac artery: A. The stent implanted in the rat aorta after surgery. B. The stent implanted in the rat aorta after 4 weeks. Arrows indicate the arterial incisions. 


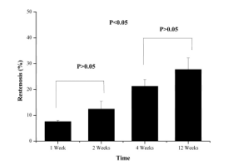

Fig. 4.

In-stent restenosis at different time points. Please note all comparisons between each two groups were significant different $(\mathrm{P}<0.05)$ other than 1 week vs. 2 weeks $(\mathrm{P}>0.05)$ and 4 weeks vs. 12 weeks $(\mathrm{P}>0.05)$. 

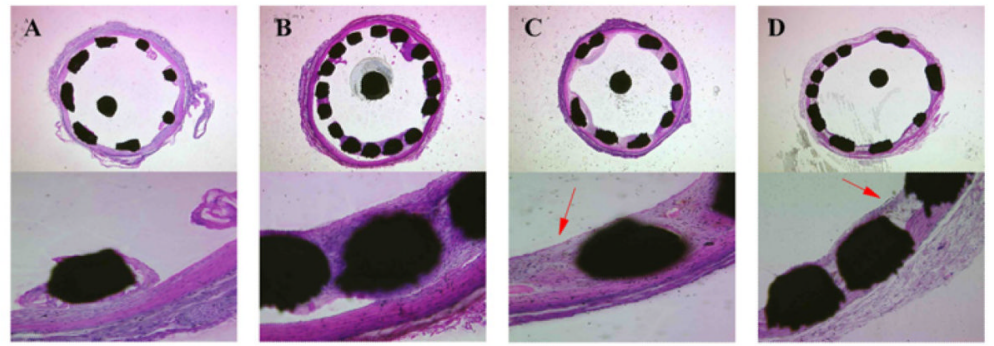

Fig. 5.

The representative images of stents at different time points post rat aorta implantation. Upper panel: 4x; Lower panel: 20x of boxed area in upper panel. A: 1 week, B: 2 weeks, C: 4 weeks, D: 12weeks. Please note no significant change in neointimal thickness between 4weeks (C) and 12 weeks (D) post implantation (arrows in C and D, low panel, and the "healed" scar "tissue at 12 weeks (D, arrow, low panel). 